\title{
Dynamic Particle Removal by Nanosecond Dry Laser Cleaning: Theory
}

\author{
N. Arnold ${ }^{1}$, G. Schrems ${ }^{2,1}$, T. Mühlberger ${ }^{1}$, M. Bertsch ${ }^{2}$, M. Mosbacher ${ }^{1,2}$, P. Leiderer $^{2}$ and D. Bäuerle \\ ${ }^{1}$ Angewandte Physik, Johannes - Kepler - Universität, A-4040 Linz, Austria \\ ${ }^{2}$ Universität Konstanz, Fachbereich Physik und Optikzentrum, Fach M676 D-78457 Konstanz, Germany \\ E-mail: nikita.arnold@jk.uni-linz.ac.at
}

\begin{abstract}
A model for ns dry laser cleaning that treats the substrate and particle expansion on a unified basis is suggested. Formulas for the time-dependent thermal expansion of the substrate, valid for temperaturedependent parameters are derived. Van der Waals adhesion, the elasticity of the substrate and particle, as well as particle inertia is taken into account for an arbitrary temporal profile of the laser pulse. Time scale related to the size of the particles and the adhesion/elastic constants is revealed. Cleaning proceeds in different regimes if the duration of the laser pulse is much shorter/longer than this characteristic time. Expressions for cleaning thresholds are provided and compared with experiments on the cleaning of $\mathrm{Si}$ surfaces from spherical $\mathrm{SiO}_{2}$ particles with radii between 200 and $2585 \mathrm{~nm}$ in vacuum with $248 \mathrm{~nm} \mathrm{KrF}$ excimer laser and $532 \mathrm{~nm}$ frequency doubled Nd-YAG laser. Large discrepancies between the experimental data and theoretical results for $\mathrm{KrF}$ laser suggest that ns dry laser cleaning cannot be explained on the basis of thermal expansion mechanism alone.
\end{abstract}

Keywords: Laser cleaning, modeling, adhesion, oscillations, $\mathrm{SiO}_{2}, \mathrm{Si}, \mathrm{LPM} 2001$.

\section{Introduction}

Laser cleaning $^{1}$ is under consideration for usage in many manufacturing processes ${ }^{2,3}$. In dry cleaning (DLC) thermal expansion is believed to be responsible for particle removal. Steam laser cleaning (SLC) ${ }^{4}$, though more efficient ${ }^{5}$, is incompatible with some applications ${ }^{6}$. Usually, one compares cleaning and adhesion forces. Nanosecond laser cleaning, due to short times involved, requires consideration of dynamic effects.

The models suggested in the literature so far require improvement. Thermal expansion of the substrate ${ }^{7}$ was treated inaccurately and separately from that of the particle $^{8,9}$. When elastic deformation of the particle compressed by the expanding substrate was considered ${ }^{10}$, adhesion and elastic forces were considered separately and the influence of the particle on the substrate deformation was not treated properly. Removal of absorbing particles and elasticity of the substrate ${ }^{11}$ was analyzed on the basis of a force balance only, without taking particle movement into account and particle temperature was estimated in a very crude way. Most of the models do not consider temporal profile of the laser pulse, which assumes infinite acceleration/deceleration. Though energy criterion was mentioned $^{12}$, its region of applicability was not clearly stated.
Redeposition and dissipative processes were considered in $^{13,14}$, but only after the pulse. Numerical results ${ }^{10}$ did not provide compact formulas for cleaning fluence. It is not clear, which parameters can improve the cleaning efficiency and decrease the cleaning threshold.

We develop an analytical model, which incorporates the influence of experimental parameters and different factors that contribute to DLC. Sound effects and field enhancement ${ }^{15,16}$ are not considered.

\section{Adhesion potential and evolution equation}

Let us introduce an approximate adhesion potential that takes into account Van der Waals (VdW) attraction and elastic forces. If a particle with radius $r$ and a plane are approached by a distance $h$ (see Fig. 1) the energy of the system is the sum of the VdW energy (work of adhesion $\varphi$ over the contact area 2 $\pi$ hr) and elastic energy, which is taken from the Hertz contact problem ${ }^{17}$. This results in the following approximate potential and force:

$U=-2 \pi \cdot h \varphi+h^{5 / 2} r^{1 / 2} \frac{2}{5 \theta}, F=2 \pi r \varphi-\frac{h^{3 / 2} r^{1 / 2}}{\theta}$

Here $\boldsymbol{\theta}$ characterizes elastic properties of the particle (p) and the substrate (s). It is dominated by the properties of the softer material (where the elastic energy is mainly stored). 


$$
\boldsymbol{\theta}=\frac{3}{4}\left(\frac{1-\sigma_{p}^{2}}{E_{p}}+\frac{1-\sigma_{s}^{2}}{E_{s}}\right)
$$

Potential (1) results in equilibrium values $h_{0}$ and $U_{0}$ :

$h_{0}=(2 \pi \varphi \theta)^{2 / 3} r^{1 / 3}$

$U_{0}=-\frac{3}{5}(2 \pi \varphi)^{5 / 3} \boldsymbol{\theta}^{2 / 3} r^{4 / 3}$

A detachment of the particle occurs when $h=0$. The maximum (pull-out) force is reached there and is given by $F_{0}=2 \pi \cdot \varphi$

This allows one to infer $\varphi$ from force measurements. The potential (1) can be approximated by a parabola of the same depth with a characteristic oscillation frequency and period:

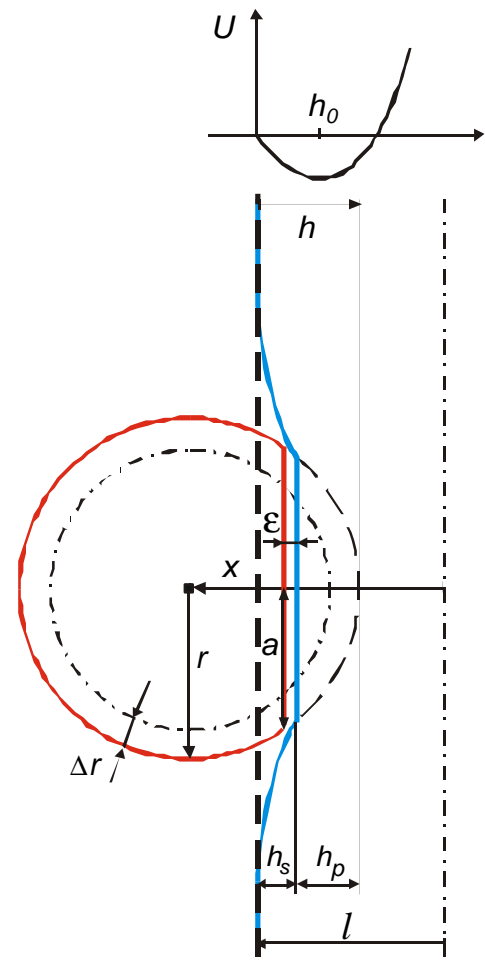

Fig. 1 Schematic of the particle-substrate deformation. Solid lines - boundaries of substrate and particles. Dashed lines -imaginary non-deformed boundaries. Dash-dotted lines -initial position of the substrate and not heated (but displaced) particle. $l$ - substrate displacement, $r$-current particle radius, $\Delta r$ - particle expansion, $x$ - position of the particle center referred to initial (non-deformed) substrate. $h$ overall deformation, $h_{p}$ and $h_{s}$ its fractions belonging to the substrate and the particle. $a$-contact radius. $\varepsilon$-equilibrium distance between adhering surfaces. Adhesion potential $U(h)$ is shown schematically.

$\boldsymbol{\omega}=\frac{3}{5^{1 / 2}(2 \pi)^{1 / 3}}\left(\frac{\boldsymbol{\varphi}}{\boldsymbol{\Theta}^{2} r^{7} \boldsymbol{\rho}_{p}^{3}}\right)^{1 / 6} \sim\left(\frac{v_{s}^{4} \boldsymbol{\varphi}}{r^{7} \boldsymbol{\rho}}\right)^{1 / 6} \sim 0.5 \times 10^{9} s^{-1}(6)$ $\tau_{0}=2 \pi / \omega \approx 10 \mathrm{~ns}$

Here $v_{s}$ is the sound velocity. Estimations assume $r \sim 1 \mu \mathrm{m}$ and material parameters from Table 1 This frequency is always lower than the frequency of the first sound mode. More realistic JKR ${ }^{18}$ potential presented in Fig. 2 is quite similar. For DMT ${ }^{19}$ case the agreement is comparable.

Equation for the evolution of $\boldsymbol{h}$. The approach $h$ is the distance over which particle and substrate are closer as compared to a point contact. Let $x$ be the coordinate of the particle center and $l$ the surface displacement in the laboratory frame, both counted from the initial position of the surface. Then

$h=l+r-x$

All quantities depend on time. By rewriting Newton equation with the force (1) for $h$ instead of $x$ with the help of (8), we obtain the equation for the evolution of $h$ (dot stands for time derivative):

$\ddot{h}+\dot{h}=\frac{1}{m}\left(2 \pi \cdot \varphi-\frac{h^{3 / 2} r^{1 / 2}}{\Theta}\right)+(\ddot{l}+\ddot{r})$

The term $m \ddot{l}$ can be interpreted as the force of inertia, but particle expansion acts in a similar way.

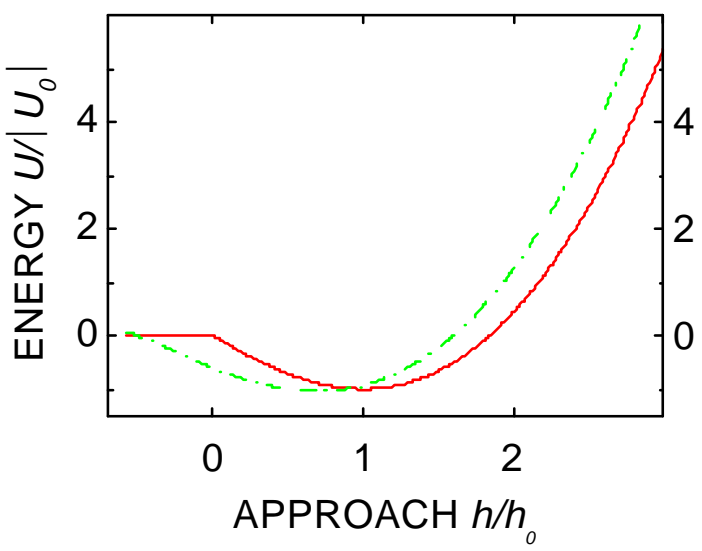

Fig. 2 Comparison between exact (dash-dotted line) and approximate (solid line) dimensionless adhesion potentials.

Damping. Damping coefficient $\gamma$ in the equation (9) can only be estimated.

Knudsen viscosity. Motion of the particle is slowed down by the presence of an ambient medium. With $r$ smaller than the mean free path of ambient gas, $\gamma$ can be estimated as:

$\boldsymbol{\gamma} \sim \frac{4 \sqrt{2}}{\pi \sqrt{\pi}} \frac{N \sqrt{m_{a} k T}}{\rho_{p} r} \sim \frac{\boldsymbol{\rho}_{g} v_{s}}{\boldsymbol{\rho}_{p} r} \sim 10^{6} s^{-1}$

Here $m_{a}$ is the mass of gas molecules and $N$ number density. Normal viscosity. With bigger particles and/or liquid layer at the surface $\gamma$ can be estimated from the Stokes formula:

$\gamma=\frac{6 \pi \eta r^{\text {gas }}}{m} \sim \frac{v_{s} r_{a}}{r^{2}} \sim 10^{5} s^{-1}$ 
where $\eta$ is the dynamic viscosity and $r_{a}$ atomic radius. This, together with thermophoresis, may be important after the detachment, i.e., for the problem of redeposition.

Absorption of sound. The rate of energy dissipation for sound waves generated by the thermal expansion that includes reflections from the boundaries yields

$$
\gamma \sim \max \left(\boldsymbol{v}, D \frac{\beta v_{s}^{2}}{c} \beta T\right) \frac{1}{d^{2}} \sim 10^{8} s^{-1}
$$

where $d \sim \min \left(r, v_{s} \tau, v_{s} / \omega, \sqrt{D \tau}\right)$ is some characteristic length, $\mathrm{v}$ and $D$ are the kinematic viscosity and thermal diffusivity of the material, $c$ its specific heat. This agrees with the logarithmic decrements of $10^{-1}-10^{-3}$ given in ${ }^{20}$.

Emission of sound by the oscillating particle into the substrate may be a primary damping mechanism. It yields:

$\gamma \approx \frac{\rho_{s}}{\rho_{p}} \frac{v_{s}}{r} \frac{1}{1+4\left(v_{s} / \omega\right)^{4}} \sim 10^{9} s^{-1}$

\section{Thermal expansion}

The results most relevant for DLC rely on classical thermoelasticity ${ }^{17}$. The derivations will be reported elsewhere. In the problem of substrate expansion there exists a hierarchy of spatial scales. Usually axial ( $z$ ) extension of the thermal field $l_{T}{ }^{3}$ and absorption length $l_{\alpha}$ are much smaller than lateral $(x-y)$ dimension of laser spot $w_{0}$, even for weakly absorbing substrates $l_{\alpha}+l_{T}<w_{0}$

If the sound does not leave the heated region in axial direction $v_{s} \tau<\left(l_{\alpha}+l_{T}\right)<w_{0}$ one has to consider dynamic unilateral expansion. With a free boundary and constant elastic parameters the surface displacement is ${ }^{21}$

$l(t)=\frac{1+\sigma}{1-\sigma} \int_{0}^{v_{s} t} \frac{\beta}{3} T\left(z, t-z / v_{s}\right) d z$

With $v_{s} \rightarrow \infty$ one recovers the static result.

If the sound leaves the heated area in axial direction, but is still within the lateral extension of the heat source, $\quad\left(l_{\alpha}+l_{T}\right)<v_{s} \tau<w_{0} \quad$ we deal with quasi-static unilateral expansion. This case is the most relevant for DLC with ns laser pulses. Here, quasi-static compressive stresses exist in $x-y$ planes. They influence the expansion in $z$ direction via the Poisson ratio $\sigma$ It is possible to bypass a solution of heat equation applying integration over $z$. For the surface displacement $l$ and surface velocity one obtains:

$l(t)=\frac{1+\sigma}{1-\sigma} \frac{\beta \phi_{a}(t)}{3 c \rho}, \quad i=\frac{1+\sigma}{1-\sigma} \frac{\beta I_{a}(t)}{3 c \rho}$

Here $\phi_{a}$ and $I_{a}$ are the absorbed fluence and intensity. Both thermal expansion and heat content within the material are proportional to the absorbed energy. This result is valid even for temperature dependent parameters if $\beta(T)$ is the differential thermal expansion coefficient, as the ratio $\beta / c \rho \approx$ const (within $10 \%$ for $\mathrm{Si}$ ) due to Grüneisen relation ${ }^{20}$.

The typical surface displacement, velocity and acceleration for a realistic excimer laser pulse

$I(t)=I_{0} \frac{t}{\tau} \exp \left(-\frac{t}{\tau}\right)$

are shown in Fig. 3. With this definition $\phi=I_{0} \tau$ and the full widths half-maximum pulse duration $\tau_{\text {FWHM }} \approx 2.45 \tau$

When the sound leaves the irradiated spot in lateral direction, $w_{0}<v_{s} \tau$ the lateral compression significantly relaxes. The elastic problem is $3 \mathrm{D}$, while heat conduction is still 1D. General results for $3 D$ quasi-static expansion with 1D heat conduction are different from the quasi-static unilateral expansion

$l(t)=2(1+\sigma) \frac{\beta \phi_{a}(t)}{3 c \rho}, \quad i=2(1+\sigma) \frac{\beta I_{a}(t)}{3 c \rho}$

Although $w_{0}$ and the spatial profile of the beam do not enter this formula explicitly, the consideration is implicitly based on the assumption that stresses and displacement disappear at infinity due to the $3 \mathrm{D}$ relaxation. Displacement at this stage is always larger than the unilateral quasi-static one, as the material as a whole is "less compressed".

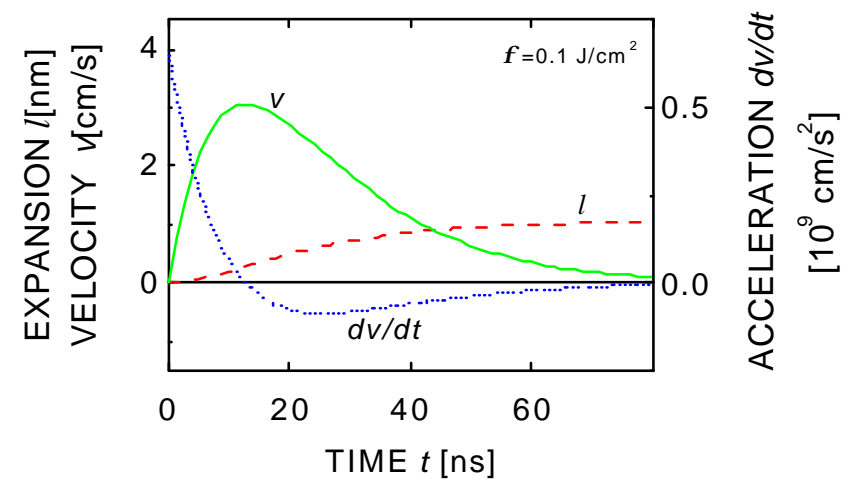

Fig. 3 Surface displacement $l$ (dashed line), velocity $v$ (solid line) and acceleration $d v / d t$ (dotted line) for a Si substrate. Laser pulse (fluence $\phi=0.1 \mathrm{~J} / \mathrm{cm}^{2}$ ) is given by (16). Other parameters are listed in Table 1.

Finally, when the heat diffuses out of the irradiated spot $w_{0}<l_{T}$, heat conduction becomes $3 \mathrm{D}$ and the heat content along the $z$ direction depends on the laser beam profile. One can obtain for the Fourier components of general $3 D$ quasistatic expansion

$\tilde{l}(\boldsymbol{k})=\frac{2 \beta(1+\boldsymbol{\sigma})}{3} \int_{0}^{\infty} e^{-k z_{1}} \tilde{T}\left(z_{1}, \boldsymbol{k}\right) d z_{1}$

This should be used with time dependent 3D temperature distributions, for example related to field enhancement by the particle $^{22}$. 
With a stationary temperature distribution and a semi-infinite substrate surface displacement is infinite everywhere as it requires infinite energy to be pumped into the system. This was overlooked in ref. ${ }^{23}$ where the expressions for displacement logarithmically diverge. Dimensionless coefficients that enter different approximations can easily differ by a factor of two.

Particle influence on the expansion of the substrate. Sometimes the thermal stress at the surface used to calculate cleaning forces is estimated ${ }^{8}$ as $\sigma_{z z} \sim \beta T E$. This stress would have existed if the substrate (particle) was not allowed to expand. The expansion, however, is restricted only by the elasticity and quite small inertia of the particle. The substrate is not appreciably slowed down by the particle. This does not mean that the substrate is not deformed. Total deformation $h$ includes particle and substrate contributions $h=h_{p}+h_{s} \quad$ (see Fig. 1), which are in relation ${ }^{17}$ $h_{p}: h_{s}=\frac{1-\sigma_{p}^{2}}{E_{p}}: \frac{1-\sigma_{s}^{2}}{E_{s}}$. Thus, "soft" substrate will have an indentation of the order of $h$, not $l$.

Thermal expansion of the particle. Temperature of a small spherical particle is homogeneous as long as $D_{p} t>>r^{2}$, i.e., with $r \sim 10^{-5} \mathrm{~cm}$ and $D_{p} \sim 0.1 \mathrm{~cm}^{2} / \mathrm{s}$, for $t>>1 \mathrm{~ns}$. The increase in its radius is given by $\dot{r}=\beta_{p} \dot{T}_{p} r / 3$, its temperature can be approximated by

$c m \dot{T}_{p}=\sigma_{a} I-4 K_{s} a\left(T_{p}-T_{s}\right)$

where $\sigma_{a} \leq \pi \sigma^{2} A$ is the absorption cross section (for small particles the expression is different), $a$ is the contact radius, and we used (8.2.10) from ${ }^{24}$ for losses into the substrate. Indices $p$ and $s$ refer to particle and substrate respectively, $T_{s}$ being the temperature of the substrate "far from the particle". Thus we obtain for an absorbing particle without thermal contact

$$
\dot{r}=\frac{\boldsymbol{\beta}_{p} r \sigma_{a}}{3 c_{p} m} I \leq \frac{\beta_{p} I_{a}}{4 c_{p} \rho_{p}}
$$

which is similar to the expression (15) for the substrate. Let us now assume that the temperature of the particle is equal to that of the substrate (ideal heat contact, upper-end estimation). Substituting an energetic estimation (7.5.8b) from $^{3}$, for the surface temperature into the expression for changes in particle radius we get for a transparent particle in thermal contact with the substrate

$$
\dot{r} \leq\left(\frac{r}{l_{\alpha}+l_{T}}\right) \frac{\boldsymbol{\beta}_{p} I_{a}}{3 c_{s} \boldsymbol{\rho}_{s}}
$$

This expression again has a structure similar to (15). The ratio $r /\left(l_{\alpha}+l_{T}\right)$ is usually less than 1 . This can be incorporated into the coefficient $C$ in (27) below.

Maximum velocity of ejected particles. Let us estimate the maximum velocity, which heavy particles can acquire. Similar estimations not recalculated into laser parameters were done in $^{13}$. Far above threshold the expansion results in a compressive elastic energy and is transformed into kinetic energy later. Energy balance (including particle expansion) yields for the ejection velocity $v$ via an elastic mechanism:

$$
\begin{aligned}
& h^{5 / 2} r^{1 / 2} \frac{2}{5 \theta}=m \frac{v^{2}}{2} \Rightarrow \\
& v \sim\left(\frac{E h^{5 / 2}}{\rho r^{5 / 2}}\right)^{1 / 2} \sim v_{s}\left(\frac{l+\Delta r}{r}\right)^{5 / 4} \sim v_{s}\left(C \frac{\beta \phi_{a}}{c \rho r}\right)^{5 / 4} \leq 10^{4} \mathrm{~cm} / \mathrm{s}
\end{aligned}
$$

Here we used (15), (20) and (27), and assumed comparable properties of particle and substrate. Usually $l, \Delta r<<r$, and the velocity of ejected particles is rather small. It is even smaller for small particles, as they move as a whole during the expansion. The velocity for the inertial mechanism cannot exceed the combined expansion velocity, which (see (27)), results in an ejection velocity $v$ :

$v \sim C \frac{\beta \boldsymbol{I}_{a}}{c \rho} \sim 0.25 \times 10^{3} \mathrm{~cm} / \mathrm{s}$

The elastic mechanism yields higher velocities. If the measured velocities exceed both estimations, other mechanisms (e.g., ablation) are involved.

\section{Cleaning threshold}

Short cleaning pulse. If laser pulse $\tau<<\tau$, one can neglect damping and adhesion force in (9) during the pulse. Then

$h(\tau) \approx h_{0}+l+\Delta r, \dot{h}(\tau) \approx 0$

and the accumulated energy is determined by changes in $h$. Cleaning will occur if this energy exceeds the total adhesion energy $\left|U_{0}\right|$. This is elastic energy cleaning regime. With potential (1) this results in

$l+\Delta r>\left((5 / 2)^{2 / 3}-1\right) h_{0} \approx 0.84 h_{0} \sim h_{0}$

where the last approximation refers to a parabolic potential.

Long cleaning pulse. If $\tau>>\tau_{\text {, }}$, oscillations are weakly excited and one can solve (9) in a quasi-static approximation. As a result, at each moment the cleaning force balances the total VdW-elastic force of adhesion. Thus, one has to overcome the adhesion force during the pulse. This is the inertial force cleaning regime. For the potential (1) the force is maximal with $h=0$ and is positive in our notations. This results in:

$-m(\ddot{l}+\ddot{r})_{\max }>F_{0}$

Thus, detachment occurs in the deceleration phase $\mathrm{e}^{7}$ due to the inertia of the already accelerated particle. The same holds for strong damping.

Dependence of the cleaning threshold on particle radius and pulse duration. Rewritten in terms of the particle radius $r$, pulse duration $\tau$ and fluence $\phi$ the conditions (25), (26) yield cleaning threshold. We combine (15) with (20) for the overall expansion of a substrate and an absorbing particle. 


$$
l+\Delta x=\left(\frac{1+\sigma_{s}}{1-\sigma_{s}} \frac{\beta_{s} A_{s}}{3 c_{s} \rho_{s}}+\frac{\beta_{p} A_{p}}{4 c_{p} \rho_{p}}\right) \phi \approx C \frac{\beta A}{c \rho} \phi
$$

For the transparent particle with thermal contact the second term will be of the order of (21), etc. In the last approximation we introduced average parameters where the material with the biggest $\beta A$ dominates, and $C \sim 0.25-1$ is dimensionless coefficient. Rewriting (25),(26) in dimensional quantities with $h_{0}$ from (3) and $\omega$ from (6) we obtain the following threshold fluences (for the pulse (16) minimal negative velocity occurs at $t=3 \pi 4)$ :

$$
\phi>C^{-1} \frac{c \rho}{\beta H}\left[\begin{array}{ll}
0.84(2 \pi \varphi \theta)^{2 / 3} r^{1 / 3} & \tau \gg>\tau_{0} \\
\frac{3}{4 \pi} \frac{\tau^{2} \varphi}{r^{2} \rho_{p}} & \tau<<\tau_{0}
\end{array}\right.
$$

Intermediate regimes can be calculated numerically. Numerical coefficients depend on the temporal profile of the laser pulse. The dependence on pulse duration is monotonous -- shorter pulses are more favorable. With $\tau<\tau_{0}$ any further decrease in pulse duration is not advantageous. With $\infty_{s} \varangle$ one has to consider sound related effects.

Fig. 4 Movement of the particle for substrate expansion

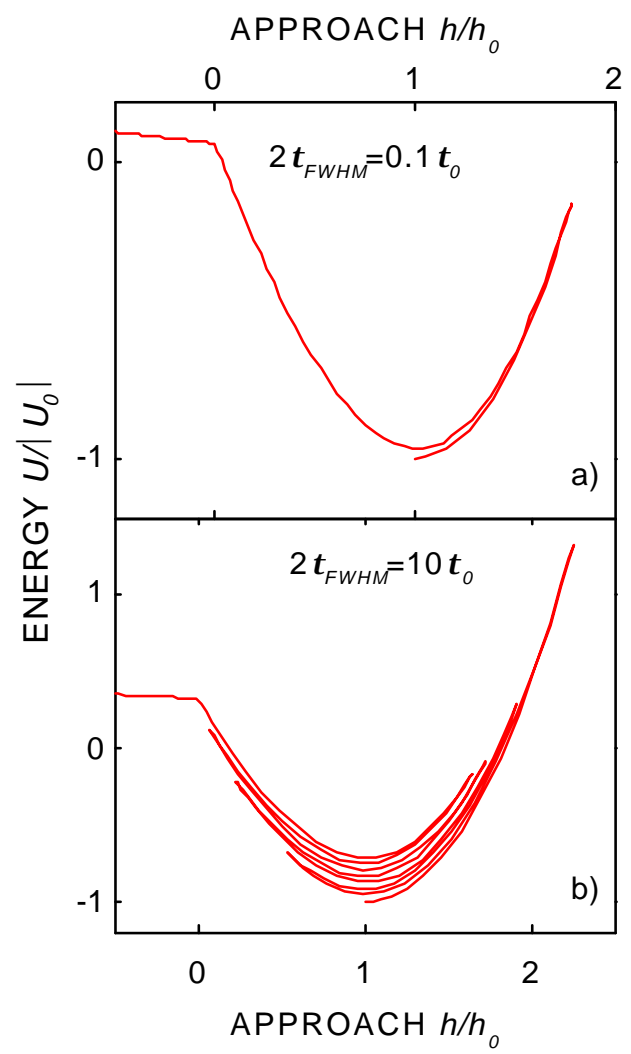

above the cleaning threshold. Laser pulse is given by (16). a) Elastic energy cleaning regime for big particle -- short pulse $2 \tau_{F W H M}=0.1 \tau_{0}$ b) Inertial force regime for small particle -- long pulse $2 \tau_{F W H M}=10 \tau_{0}$.
The dependence on the particle radius is non-monotonous. There exists an optimal radius for a given pulse duration with $\tau_{0}(r) \sim \tau$ With bigger radii the period $\tau_{0}(r)>>\tau$ the cleaning force is much shorter than the oscillation cycle and cleaning proceeds in the "elastic energy" regime. Heavy particle almost does not move during the pulse hence the substrate surface moves much faster than the center of the particle. This leads to an increase in elastic energy (compression of substrate and particle) and detachment after the end of the pulse. Detachment occurs in the first backward swing of the oscillation. This is shown in Fig. 4a. From the physical point of view, $r^{1 / 3}$ increase in threshold is due to bigger equilibrium $h_{0}$ and higher overall adhesion energy (4) for larger particles. With smaller particles $\tau_{0}(r)<<\tau$ the response of the oscillator to the "low frequency" force is inefficient. Cleaning proceeds in the "quasi-static" regime, with small fast oscillations in $h$ superimposed on the slow "drift" in $h$ that obeys force balance. This regime is shown in Fig. 4b. Strong increase in threshold for smaller particles demonstrates inefficiency of "inertial force" cleaning regime. Figure 5 shows the movement of the particle for $\tau \tau_{0}$ when no simple approximations exists. Initially surface displacement $l$ is faster than the particle movement (compression) and later the particle detaches with constant velocity.

Fig. 5 Movement of the particle slightly above cleaning threshold. Potential and pulse shape as in Fig. 4 with

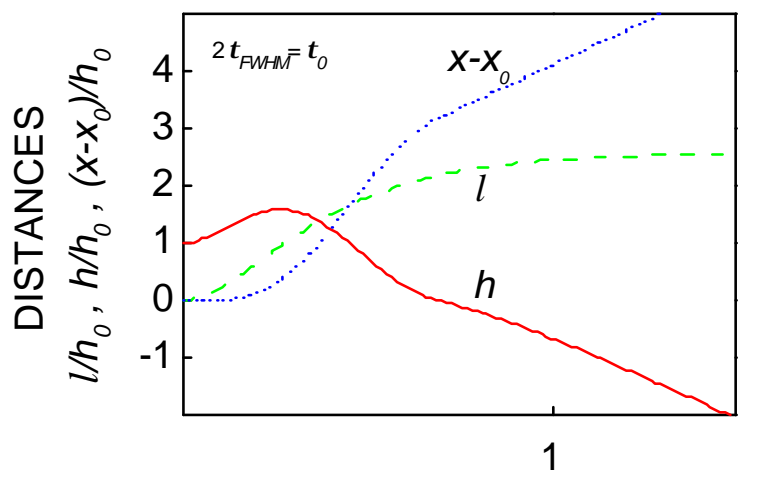

TIME $t / \tau_{0}$

$2 \tau_{F W H M}=\tau_{0}$. Solid line - evolution of the approach $h$. Dashed line - surface expansion $l$. Dotted line - particle center $x$ in the laboratory reference frame referred to its initial position $x_{0}$.

\section{Example of $\mathrm{SiO}_{2}$ particles cleaned from $\mathrm{Si}$ wafers}

Experimental. Cleaning of $\mathrm{SiO}_{2}$ particles (radii 200-2585 nm) from (100) Si wafers was studied with $\mathrm{KrF}$ excimer laser (248 $\mathrm{nm}$, pulse duration $31 \mathrm{~ns}$ FWHM) and frequency doubled Nd-YAG laser (532 nm, $7 \mathrm{~ns}$ FWHM) radiation. The laser spot on the target has a circular top-hat profile with $1 \mathrm{~mm}$ diameter. The sample was fixed in a vacuum chamber $\left(4 \times 10^{-5}\right.$ 
mbar) to minimize humidity and redeposition. Particles were deposited by spin-coating. Optical microscopy and image processing software were used to evaluate the cleaning efficiency. Pictures of the cleaned area before and after irradiation were taken and compared to monitor the behavior of clusters and particle redeposition.

Figure 6 compares theoretical and experimental results. Employed parameters are listed in Table 1. Their choice is not unambiguous. $\mathrm{Si}$ is anisotropic, vitreous $\mathrm{SiO}_{2}$ does not accurately follow the Grüneisen relation, etc. Absorption and expansion of fused silica are much smaller than that of $\mathrm{Si}$, and were neglected, as well as damping. In the presence of native oxide, the contact is $\mathrm{SiO}_{2}-\mathrm{SiO}_{2}$, which reduces the work of adhesion $\varphi$ This value was used in the calculation for the Nd-YAG laser. The surface roughness and residual moisture can further change adhesion. Having this in mind we also present calculations for 10 times smaller $\varphi$ Though the cleaning threshold increases with decreasing particle size with both the excimer laser (circles) and Nd-YAG laser (triangles) data, the agreement with the theoretical results is quite different. With the KrF-laser data both the observed values and the slope of $\phi_{l}=\phi_{l}(r)$ are much lower than predicted, while for Nd-YAG laser radiation the agreement is reasonable.

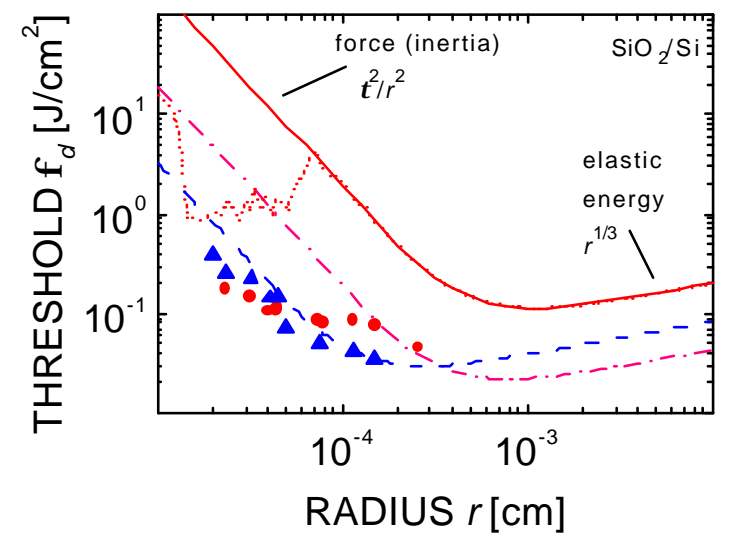

Fig. 6 Experimental (symbols) and calculated cleaning threshold fluence as a function of particle radius for $\mathrm{SiO}_{2}$ particles on Si. Circles - KrF laser, triangles - Nd-YAG laser. Solid line - calculated threshold for KrF laser. Dash-dotted line - threshold for 10 times smaller adhesion. Dashed line threshold for Nd-YAG laser. Dotted line - threshold for "rigged" KrF laser pulse.

Table 1. Parameters used in the calculations.

\begin{tabular}{|l|l|}
\hline Pulse duration $\tau($ see $(16))$ & $\begin{array}{l}12.7 \mathrm{~ns}(\mathrm{KrF}) \\
2.86 \mathrm{~ns}(\mathrm{Nd}-\mathrm{YAG})\end{array}$ \\
\hline Substrate $\boldsymbol{S i}$ & \\
\hline Specific heat $c_{s}$ & $0.72 \mathrm{~J} / \mathrm{gK}$ \\
\hline Volumetric thermal expansion $\beta$ & $7.7 \times 10^{-6} \mathrm{~K}^{-1}$ \\
\hline
\end{tabular}

\begin{tabular}{|l|l|}
\hline Poisson ratio $\sigma$ & 0.27 \\
\hline Young modulus $E_{s}$ & $1.6 \times 10^{12} \mathrm{dynes} / \mathrm{cm}^{2}$ \\
\hline Density $\rho_{s}$ & $2.3 \mathrm{~g} / \mathrm{cm}^{3}$ \\
\hline Absorption coefficient $\alpha_{s}$ & $1.67 \times 10^{6} \mathrm{~cm}^{-1}(\mathrm{KrF})$ \\
& $9 \times 10^{3} \mathrm{~cm}^{-1}(\mathrm{Nd}-\mathrm{YAG})$ \\
\hline Absorptivity $A_{s}$ & $0.39(\mathrm{KrF})$ \\
& $0.63(\mathrm{Nd}-\mathrm{YAG})$ \\
\hline Particle $\mathrm{SiO}_{2}$ & \\
\hline Specific heat $c_{p}$ & $1 \mathrm{~J} / \mathrm{gK}$ \\
\hline Volumetric thermal expansion $\beta_{p}$ & $1.65 \times 10^{-6} \mathrm{~K}^{-1}$ \\
\hline Poisson ratio $\sigma_{p}$ & 0.17 \\
\hline Young modulus $E_{p}$ & $0.73 \times 10^{12} \mathrm{dynes} / \mathrm{cm}^{2}$ \\
\hline Density $\boldsymbol{P}_{p}$ & $2.2 \mathrm{~g} / \mathrm{cm}^{3}$ \\
\hline Work of adhesion $\varphi_{\mathrm{Si}} \mathrm{SiO}_{2}$ & $140 \mathrm{erg} / \mathrm{cm}^{2}(\mathrm{KrF})$ \\
$\mathrm{SiO}_{2}-\mathrm{SiO}_{2}$ & $76 \mathrm{erg} / \mathrm{cm}^{2}(\mathrm{Nd}-\mathrm{YAG})$ \\
\hline
\end{tabular}

One possible explanation for this discrepancy is the "bad quality" of the excimer laser pulse. It is too long for small particles and they are removed in the inefficient "force" regime. Spikes in intensity, when in resonance with $\tau_{0}$, may strongly decrease the threshold. Calculations for the rigged pulse with angular frequencies up to $\sim 30 / \tau$ are shown by the dotted line in Fig. 6. The surface displacement is almost the same as for the smooth pulse (Fig. 3), but the velocity and especially the acceleration differs significantly (Fig. 7). Another reason may be the effect of field enhancement by the particles ${ }^{15,22}$.

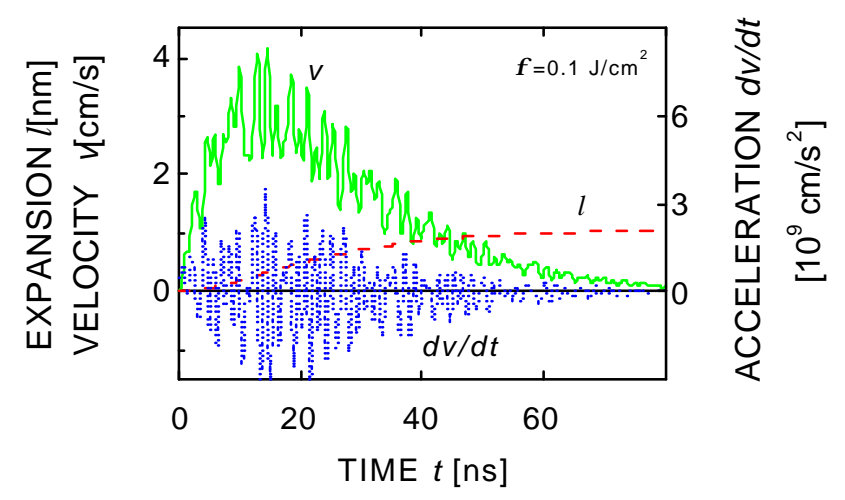

Fig. 7 Surface displacement $l$ (dashed line), velocity $v$ (solid line) and acceleration $d v / d t$ (dotted line) for the rigged pulse (dashed line in Fig. 6). All other parameters are as in Fig. 3. Note the difference in scale for acceleration as compared to Fig. 3.

\section{Experimental suggestions}

One can try to utilize resonance effects to remove smaller particles with the same fluence but lower heating.

A ns excimer laser pulse is "too long" for cleaning sub- $\mu \mathrm{m}$ particles. If it is modulated with the frequency that 
matches the "adhesion frequency" 6), one can expect a resonance increase in oscillation amplitude. Calculations show that if the total duration of a pulse stays constant, and the period of oscillations is about 0.1 of the overall pulse duration, the cleaning threshold can be decreased by 1-2 orders of magnitude. With a non-linear potential (1) resonant growth saturates after 5-10 oscillations.

Without damping there is no difference between the cleaning by the single resonant pulse $\tau=\tau_{0}$ and longer modulated pulse with $n$ resonant "pushes" $\tau=n \tau_{\text {, with the }}$ same total fluence. The damage threshold, however, will decrease for the longer pulse. In this way one can increase the window for damage-free cleaning.

Shorter pulses are more efficient. With ps pulses, the damage threshold is determined by $l_{\alpha}$ and is much lower than with ns pulses. One can replace a single ps pulse by several pulses with a fixed delay $\tau_{0}$ in between. Mode locked lasers are natural candidates for such experiments. Cleaning will be comparable, while damage will be determined by the overall duration $n \tau_{0}$ of the train of $n$ pulses, provided that $l_{T} \sim\left(D_{s} n \tau_{0}\right)^{1 / 2}>>l_{\alpha}$.

\section{Conclusions}

We formulated the DLC problem with ns pulses as an escape from the adhesion potential under the action of a cleaning force related to thermal expansion. Model expressions for this potential and damping forces are suggested. Thermal expansion of the substrate and the particle are treated on a unified basis. Expressions for thermal expansion that do not require solution of the heat equation and are valid for temperature-dependent parameters are discussed.

In addition to the pull-out force $F_{0}$, parameters of the adhesion potential most important for DLC are the period of oscillations $\tau_{0}$ and equilibrium deformation $h_{0}$. Their dependence on particle size $r$ and material properties is provided. The laser pulse duration $\tau$ should be compared with $\tau_{0}$, and the overall thermal expansion $l+\Delta r$ with $h_{0}$. Simple formulas for the cleaning threshold $\phi_{l}$ are derived. With $\tau<\tau_{0}$ (big particles) cleaning takes place in the "elastic energy" regime, which requires $l+\Delta r>h_{0}$ resulting in $\phi_{l l} \propto r^{1 / 3}$. With $\tau>\tau_{0}$ (small particles) cleaning occurs in the "inertial force" regime, which requires decelerations $-m(\ddot{l}+\ddot{r})_{\max }>F_{0}$, leading to $\phi_{e} \propto \tau^{2} / r^{2}$.

Thermal expansion mechanism cannot fully explain experiments with $\mathrm{SiO}_{2}$ particles on $\mathrm{Si}$ surfaces. Thresholds observed in experiments with $\mathrm{KrF}$ lasers are too low. Possible explanations are fast spatial-temporal variations in intensity of excimer pulses and field enhancement effects. Utilization of resonance effects by modulation of ns laser pulses or by employing ps pulses with delays equal to $\tau_{b}(r)$ is suggested.
Acknowledgments. We thank Prof. B. Luk'yanchuk, HD Dr. J. Boneberg and Dr. B.-U. Runge for useful discussions. Financial support by the EU TMR project Laser Cleaning $n^{\circ}$ ERBFMRXCT98 0188, and the Fonds zur Förderung der wissenschaftlichen Forschung in Österreich project P14700TPH is gratefully acknowledged.

\section{References}

${ }^{1}$ W. Zapka, W. Ziemlich, and A. C. Tam, Appl. Phys. Lett. 58 2217 (1991)

${ }^{2}$ The national Technology Roadmap for Semiconductors (Semic. Industry Association), San Jose, CA, 116 (1994)

3 D. Bäuerle: Laser Processing and Chemistry, $3^{\text {rd }}$ ed., Springer, Berlin, 2000

${ }^{4}$ S.J.Lee, K.Imen, S.D.Allen Appl.Phys.Lett. 58203 (1991)

${ }^{5}$ M. She, D. Kim and C. P.Grigoropoulos J. Appl. Phys., 86 6519 (1999)

${ }^{6}$ R.D. Halfpenny, D.M. Kane, J.Appl.Phys. 866641 (1999)

${ }^{7}$ V. Dobler, R. Oltra, J.P. Boquillon, M. Mosbacher, J. Boneberg, and P. Leiderer, Appl. Phys. A, 69335 (1999)

${ }^{8}$ Y. F. Lu, W. D. Song, B. W. Ang, M. H. Hong, D. S. H. Chan, T. S. Low, Appl. Phys. A, 659 (1997)

${ }^{9}$ V. P. Veiko, E. A. Shakhno, in Proc. SPIE, (2001)

${ }^{10}$ Y. F. Lu, Y. W. Zheng and W. D. Song, Appl. Phys. A, 68 569 (1999)

${ }^{11}$ Y. F. Lu, W. D. Song, M. H. Hong, B. S. Teo, T. C. Chong and T. S. Low, J. Appl. Phys., 80499 (1996)

12 Al.A. Kolomenskii, H. A. Schuessler, V. G. Mikhalevich and A. A. Maznev, J. Appl. Phys., 842404 (1998)

${ }^{13}$ Y. F. Lu, Y. W. Zheng, and W. D. Song, J. Appl. Phys., 87 549 (2000)

${ }^{14}$ G. Vereecke, E. Röhr, and M. M. Heyns, J. Appl. Phys., 85 3837 (1999)

${ }^{15}$ P. Leiderer, J. Boneberg, V. Dobler, M. Mosbacher, H.-J. Münzer, T. Fourrier, G. Schrems, D. Bäuerle, J. Siegel, N. Chaoui, J. Solis, C. N. Afonso, Proc. SPIE 4065249 (2000)

${ }^{16}$ Y. F. Lu, Y. W. Zheng, and W. D. Song, J. Appl. Phys., 87 $1534(2000)$

${ }^{17}$ L. D. Landau and E. M. Lifshitz, Theory of elasticity, Pergamon Press, New York, 1986, §§6,7

${ }^{18}$ K. L. Johnson, K. Kendall, and A. D. Roberts, Proc. R. Soc. London Ser. A., 324301 (1971)

${ }^{19}$ B. V. Derjagin, V. M. Muller, and Yu. P. Toropov, J. Colloid Interface Sci., 53314 (1975)

${ }^{20}$ D. E. Gray (ed.), American Institute of Physics Handbook, McGraw-Hill, New York, 1972

${ }^{21}$ A.A. Maznev, J. Hohlfeld, J. Güdde, J.Appl.Phys., 825082 (1997)

${ }^{22}$ B. Luk'yanchuk, Y.W. Zheng, Y.F. Lu, Proc. SPIE, 4065 576 (2000) 
${ }^{23}$ L. P. Welsh, J. A. Tuchman, I. P. Herman, J. Appl. Phys., 64 6274 (1988)

${ }^{24}$ H.S. Carslaw and J.C. Jaeger, Conduction of Heat in Solids, Oxford Univ. Press, New York, 1959 